\title{
Executive Summary: Clinical Practice Guideline for Autosomal Dominant Polycystic Kidney Disease in China
}

\author{
Chang-Lin Mei ${ }^{\mathrm{a}}$ Cheng Xue ${ }^{\mathrm{a}}$ Sheng-Qiang Yu${ }^{\mathrm{a}}$ Bing Dai ${ }^{\mathrm{a}} \quad$ Jiang-Hua Chen ${ }^{\mathrm{b}}$ Ying $\mathrm{Li}^{\mathrm{c}}$ \\ Li-Meng Chen $^{d}$ Zhang-Suo Liu ${ }^{e}$ Yong-Gui Wu ${ }^{f}$ Zhao Hu ${ }^{g}$ Yan Zhah Hong Liu ${ }^{\mathrm{i}}$ \\ Yong-Ze Zhuang ${ }^{j}$ Chun Zhang ${ }^{k}$ Xiang-Cheng Xiao' Yue Wang ${ }^{\mathrm{m}}$ Gui-Sen Li ${ }^{\mathrm{n}}$ Yi-Yi Ma $^{\mathrm{a}}$ \\ Lin $\mathrm{Li}^{\mathrm{a}}$ ADPKD Clinical Guideline Working Group \\ ${ }^{a}$ Department of Nephrology, Changzheng Hospital, Second Military Medical University, Shanghai, China; \\ ${ }^{b}$ Department of Nephrology, The First Affiliated Hospital, Zhejiang University School of Medicine, Hangzhou, \\ China; ' Department of Nephrology, The Third Affiliated Hospital, Hebei Medical University, Shijiazhuang, China;

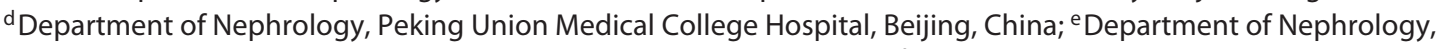 \\ The First Affiliated Hospital, Zhengzhou University, Zhengzhou, China; ${ }^{\mathrm{f} D e p a r t m e n t}$ of Nephrology, The First \\ Affiliated Hospital, Anhui Medical University, Hefei, China; ${ }^{9}$ Department of Nephrology, Qilu Hospital, Shandong \\ University, Jinan, China; ${ }^{h}$ Department of Nephrology, Guizhou Provincial People's Hospital, Gui Yang, China; \\ 'Department of Nephrology, Second Xiangya Hospital, Central South University, Changsha, China; 'Department \\ of Nephrology, 900 Hospital of the Joint Logistics Team, PLA, Fuzhou General Clinical Medical College of Fujian \\ Medical University, Fuzhou, China; ' Department of Nephrology, Union Hospital, Tongji Medical College, Huazhong \\ University of Science and Technology, Wuhan, China; 'Department of Nephrology, Xiangya Hospital, Central

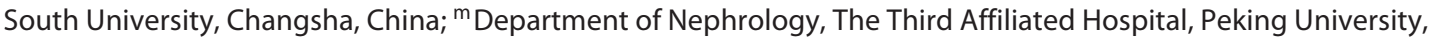 \\ Peking, China; ${ }^{n}$ Sichuan Academy of Medical Science and Sichuan Provincial People's Hospital, School of Medicine, \\ University of Electronic Science, Chengdu, China
}

\section{Keywords}

Autosomal dominant polycystic kidney disease $\cdot$ Gene .

Diagnosis · Treatment · End-stage renal disease

\begin{abstract}
Autosomal dominant polycystic kidney disease (ADPKD) is the most common hereditary kidney disease, with a prevalence of 1/2,500-1/1,000, and it affects 1.25 million people in China. ADPKD is responsible for nearly $5 \%$ of end-stage renal disease cases, which leads to a major burden on public health. In 2016, the Chinese working group developed guidelines for the diagnosis and treatment of ADPKD, which
\end{abstract}

promoted the clinical management of ADPKD in China. In the last 3 years, Chinese clinicians have deepened their understanding and standardized the management of ADPKD, and several basic and clinical studies on ADPKD have been conducted. In combination with international guidelines and research results, the working group updated the ADPKD guidelines in China. This guideline includes 5 chapters: introduction, diagnosis, kidney disease progression monitoring, treatment, and family planning. We highlight the main recommendations and suggestions of the ADPKD guidelines in this summary.

(C) 2020 The Author(s)

Published by S. Karger AG, Basel

\begin{tabular}{ll}
\hline karger@karger.com & (c) 2020 The Author(s) \\
Published by S. Karger AG, Basel & Karger \\
Thw.karger.com/kdd & This article is licensed under the Creative Commons Attribution- \\
NonCommercial-NoDerivatives 4.0 International License (CC BY- \\
NC-ND) (http://www.karger.com/Services/OpenAccessLicense). \\
Usage and distribution for commercial purposes as well as any dis- \\
tribution of modified material requires written permission.
\end{tabular}

Chang-Lin Mei or Cheng Xue

Kidney Institute, Department of Nephrology

Changzheng Hospital

415 Fengyang Rd., Shanghai 200003 (China)

changlinmei@smmu.edu.cn or xuecheng@smmu.edu.cn 


\section{Chapter 1: Introduction}

Autosomal dominant polycystic kidney disease (ADPKD) is the most common hereditary nephropathy, with a prevalence of $1 / 2,500-1 / 1,000$, and it affects 1.25 million people in China. ADPKD is responsible for nearly $5 \%$ of end-stage renal disease cases. Approximately half of the patients progress to ESRD by 60 years of age, which leads to a major burden on public health. It is characterized by the continuous development and growth of renal cysts causing relentless kidney enlargement associated with hypertension, abdominal pain, cyst hemorrhage, gross hematuria or infection, nephrolithiasis, a reduced quality of life, and gradually damaged renal structure and function with aging. As a systemic disorder, ADPKD often affects multiple organs (e.g., polycystic liver disease $[\mathrm{PLD}]$, intracranial aneurysms, and abnormal heart valves).

There are 2 overwhelming pathogenic mutations in ADPKD, i.e., $P K D 1$ and $P K D 2$. Diseases caused by $P K D 1$ and $P K D 2$ mutations account for approximately 78 and $15 \%$ of the total cases of ADPKD, respectively. There are possibly other PKD genes, such as GANAB and DNAJB11. Patients with $P K D 2$ mutations have milder kidney disease with fewer renal cysts, a delayed onset of hypertension and ESRD, and a longer survival compared to those with PKD1 mutations. Moreover, truncating PKD1 mutations are associated with more severe kidney disease compared to nontruncating PKD1 mutations.

Based on the clinical characteristics, diagnosis, and treatment practices, the Chinese ADPKD Clinical Guideline Working Group summarizes the consensus on ADPKD.

\section{Chapter 2: Diagnosis}

\subsection{Imaging Approaches for Diagnosis}

2.1.1 We recommend ultrasound as the first-line imaging approach for ADPKD diagnosis. (1B)

2.1.2 We suggest using ultrasound-based unified criteria for the diagnosis or exclusion of ADPKD in at-risk cases with a positive family history (Table 1). (2B)

2.1.3 We suggest magnetic resonance imaging (MRI) as an alternative imaging modality for patients with an equivocal ultrasound diagnosis or when genotyping tests cannot be performed. (2B)

2.1.4 We suggest using the MRI-based criteria for the diagnosis or exclusion of ADPKD in at-risk cases with a positive family history (Table 2). (2B)

Executive Summary: Clinical Practice

Guideline for ADPKD in China
2.1.5 For individuals with imaging findings of renal cysts and without a positive family history, the following factors should be considered when making the diagnosis: (not graded)

1. Ultrasound should be carried out on the affected patient's parents to assess asymptomatic PKD.

2 Ultrasound for extrarenal cystic diseases, including liver and pancreas, can help with diagnosis.

3. For equivocal or atypical cases, serial imaging to track cyst growth or genotyping testing may be necessary to make the diagnosis of ADPKD.

4. An arbitrary number of 10 renal cysts detected by ultrasound could be the general consensus for clinical diagnosis.

\subsection{Genetic Testing}

2.2.1 We recommend that the standard method for the genetic diagnosis of ADPKD is long-sequence polymerase chain reaction combined with next-generation sequencing technology, and Sanger sequencing is used for confirmation. (1B)

2.2.2 Genetic testing should be performed in the following situations: (2D)

1. Patients without a positive family history of ADPKD: $10-15 \%$ of ADPKD patients do not have a positive family history, such as sporadic ADPKD or missing parental data.

2. Patients with a positive family history of ADPKD are potential kidney donors, but ultrasound examination cannot confirm the diagnosis of ADPKD.

3. Specific types of ADPKD (such as early and severe ADPKD, obvious asymmetry of renal cysts, atypical imaging findings, renal failure without significant renal enlargement, and significantly different disease conditions among family members).

4. Reproductive counselling or preimplantation genetic testing is needed. The PKD mutation detection rate is approximately 85 and $15 \%$ of patients with suspected ADPKD are still undetectable.

\subsubsection{Genetic counselling}

We recommend that patients diagnosed with ADPKD or individuals at risk of ADPKD be referred to genetics services for genetic counselling if they would like to discuss the following: (2B)

1 inheritance pattern and clarification of their ADPKD risk for themselves or their family members

2 indications, role, and interpretation of molecular genetic testing

3 family plan and preimplantation genetic testing. 
Table 1. Ultrasound-based criterion for the diagnosis or exclusion of ADPKD

\begin{tabular}{llll}
\hline Age, years & $15-39$ & $40-59$ & $>60$ \\
\hline $\begin{array}{l}\text { Diagnostic confirmation } \\
\text { Disease exclusion }\end{array}$ & $\begin{array}{l}\text { At least 3 cysts (unilateral or bilateral) } \\
\text { No recommendation }\end{array}$ & $\begin{array}{l}\text { At least 2 cysts in each kidney } \\
<2 \text { cysts in each kidney }\end{array}$ & $\begin{array}{l}\text { At least } 4 \text { cysts in each kidney } \\
<2 \text { cysts in each kidney }\end{array}$ \\
\hline
\end{tabular}

Table 2. Suggested MRI criterion for diagnosis or exclusion of ADPKD

\begin{tabular}{ll}
\hline ADPKD & Age $15-39$ years \\
\hline Diagnosis & $>10$ cysts in total \\
Exclusion & $<5$ cysts in total \\
\hline
\end{tabular}

\section{Chapter 3: Kidney Disease Progression Monitoring}

3.1 We recommend that the height-adjusted total kidney volume (HtTKV) be used for the initial evaluation of patients with ADPKD. Repeated TKV measurements in asymptomatic patients with ADPKD without intervention to slow disease progression are not suggested. Repeated imaging is suggested when disease-modifying treatments or lifestyle modifications are shown to alter ADPKD progression. (not graded)

3.5 We suggest using MRI T2-weighted imaging to measure TKV by the ellipsoid equation based on the orthogonal length, width, and thickness of the kidney. Imaging reports should be standardized. (not graded)

The ellipsoid equation is: TKV for each kidney $=\pi \times 1$ $\times \mathrm{w} \times \mathrm{t} / 6$, where $\mathrm{l}=$ length, $\mathrm{t}=$ thickness, and $\mathrm{w}=$ width . The Mayo Clinic Imaging Classification facilitates TKV use. According to the classification, ADPKD can be divided into 2 categories, of which type 1 is the typical imaging manifestation of ADPKD, accounting for approximately $95 \%$ of all cases; type 2 is atypical imaging manifestations. HtTKV is used to classify the progression of type 1 patients into 5 subtypes, i.e., $1 \mathrm{~A}, 1 \mathrm{~B}, 1 \mathrm{C}, 1 \mathrm{D}$, and $1 \mathrm{E}$, estimated from a starting HtTKV $(150 \mathrm{~mL} / \mathrm{m})$; the corresponding TKV annual growth rate for each subtype is $<1.5,1.5-3,3-4.5,4.5-6$, and $>6 \%$, respectively. A model based on this classification can predict future GFR decline (http://www.mayo.edu/research/documents/pkdcenter-adpkd-classification/doc-20094754).

3.1 We suggest using the estimated glomerular filtration rate (eGFR) to monitor ADPKD progression. (2B)

3.2 We suggest using the Chronic Kidney Disease Epidemiology Collaboration (CKD-EPI) equation to calcu- late the eGFR when monitoring renal function in patients with ADPKD. (2B)

3.3 We suggest using proteinuria or albuminuria to monitor ADPKD progression. (2B)

Approximately $25 \%$ of patients diagnosed with ADPKD have proteinuria ( $>300 \mathrm{mg} /$ day, but it usually does not exceed $1 \mathrm{~g}$ /day). Proteinuria is associated with a faster decline in eGFR and earlier onset of ESRD. Proteinuria in the nephrotic range might indicate an additive disorder.

\section{Chapter 4: Treatment}

\subsection{Diet and Lifestyle Management}

4.1.1 We recommend that patients with ADPKD should restrict their sodium intake (daily sodium intake $2.3-3$ or $<6$ g salt) adjusted for extrarenal losses (hot climate, sauna, runners, and bowel disease) if appropriate. (1C)

4.1.2 We recommend that patients with ADPKD consume a moderate-protein diet $(0.8-1.0 \mathrm{~g} / \mathrm{kg}$ of ideal body weight). (1C)

Monitor protein intake: $6.253 \times$ (urine urea nitrogen [g/day] $+0.033 \times$ weight $[\mathrm{kg}])$.

4.1.3 We recommend that patients with ADPKD maintain plasma bicarbonate within the normal range (>22 mmol/L). (2B)

Vegetable and fruit intake should increase if the plasma bicarbonate level is $<22 \mathrm{mmol} / \mathrm{L}$. Oral sodium bicarbonate should be taken if needed.

4.1.4 We recommend that patients with ADPKD maintain a normal BMI with moderation of the caloric intake. (2B)

4.1.5 We recommend that patients with ADPKD control their lipid intake to maintain serum LDL $\leq 100 \mathrm{mg} /$ dL. (2B)

Regular exercise should be performed, and statins can be taken if needed. Ezetimibe can be used if patients are intolerant of statins.

4.1.6 We suggest that patients with ADPKD moderately enhance their water intake to maintain urine osmo- 
lality $\leq 280 \mathrm{mOsm} / \mathrm{kg}$. The first morning urine osmolality should be monitored if available. (2C)

Water prescription $(\mathrm{L})=24$-h urine osmolality $/ 280+$ unmeasurable loss of water $\left(\int 0.5 \mathrm{~L}\right)$.

4.1.7 We suggest a moderate phosphate restriction diet ( $\leq 800 \mathrm{mg}$ /day). (2C)

Read labels of food and watch for additives containing phosphates. The use of phosphate binders is not different from other CKD when needed.

4.1.8 We recommend that patients with ADPKD maintain serum uric acid within the normal range ( $\leq 7$ $\mathrm{mg} / \mathrm{dL}$ for males; $\leq 6 \mathrm{mg} / \mathrm{dL}$ for females). (2B)

Oral sodium bicarbonate or febuxostat can be taken if needed.

4.1.9 We suggest that patients with ADPKD receive psychosocial support and counselling to address selfmanagement, psychological issues, social challenges, and education if needed. (2D)

\subsection{Hypertension}

4.2.1 We recommend the use of antihypertensive treatments to control hypertension with a targeted blood pressure $\leq 130 / 80 \mathrm{~mm} \mathrm{Hg}$ in patients with ADPKD. (1B)

4.2.2 We recommend the use of antihypertensive treatments to control hypertension with a targeted blood pressure $\leq 110 / 75 \mathrm{~mm} \mathrm{Hg}$ in patients with ADPKD with an eGFR $>60 \mathrm{~mL} / \mathrm{min} / 1.73 \mathrm{~m}^{2}, 18-50$ years old, and with Mayo Clinic class 1C-1E or intracranial aneurysms. (1B)

4.2.3 We recommend angiotensin-converting enzyme inhibitors (ACEI) or angiotensin receptor blockers (ARB) as first-line antihypertensive therapy. (1B)

We do not suggest dual renin-angiotensin blockade. (1B)

If intolerant, $\alpha / \beta$ blockers, cardioselective $\beta$-blockers, dihydropyridine calcium channel blockers, and diuretics are considered second-line antihypertensive therapies. (1C)

4.2.4 We recommend that children with a family history of ADPKD be screened for hypertension from age 5 years onward, with an interval of 3 years in children with no hypertension found. ACEI/ARB is preferred as the first-line treatment. (not graded)

\subsection{Disease-Modifying Treatments for High-Risk ADPKD Patients}

4.3.1 We recommend V2 receptor antagonists (tolvap$\tan$ ) for patients with ADPKD with Mayo Clinic class 1C, $1 \mathrm{D}$, or $1 \mathrm{E}$ and $\mathrm{CKD}$ stages $1-3$. (1B)

Executive Summary: Clinical Practice

Guideline for ADPKD in China
Tolvaptan, a vasopressin V2 receptor antagonist, can effectively inhibit renal cyst growth in patients with ADPKD and delay renal function deterioration. The major side effects of tolvaptan treatment include hepatotoxicity, polyuria, pollakiuria, nocturia, thirst, and fatigue. Doses of tolvaptan need to be split with the first taken early in the morning and the second taken eight hours later in the afternoon. A 45-mg dose in the morning and $15-\mathrm{mg}$ dose in the afternoon were given initially and then titrated to $60 / 30$ and $90 / 30 \mathrm{mg}$ in $15-\mathrm{mg}$ increments every 1-2 weeks if tolerated or until the urine osmolality was $\leq 280 \mathrm{mOsm} / \mathrm{kg}$ before the morning dose. Plasma sodium should be optimally maintained at $135-143 \mathrm{mmol} / \mathrm{L}$. Pregnancy, uncorrected hypernatremia, lactation, hypovolemia, a history of severe liver injury not caused by PLD, urinary tract obstruction, concomitant use of strong CYP3A inhibitors, and inability to sense thirst are contraindications.

4.3.2 In consideration of the potential hepatocellular toxicity, we suggest monitoring liver function before tolvaptan initiation and after 2 and 4 weeks, then monthly for 18 months, and every 3 months thereafter. (2B)

4.3.3 We do not recommend yearly TKV measurements to monitor tolvaptan's efficacy. (2B)

Monitoring of the yearly rate of eGFR decline or measuring TKV every 3-5 years compared with the initial data can be used for reassurance of tolvaptan treatment.

4.3.4 We suggest that patients with ADPKD on tolvaptan treatment ingest water in anticipation of thirst to avoid dehydration; ingest at least 2-3 L of water during the day and 1-2 cups of additional water before bedtime, regardless of perceived thirst; and replenish water after each episode of nocturia. (not graded)

\subsection{Treatment of Complications}

4.4.1 Hematuria and Cyst Hemorrhage

1. We suggest that antifibrinolytic drugs, such as ranexamic acid, be used for patients with ADPKD with persistent gross hematuria or cyst hemorrhage (more than 1 week), and the prophylactic use of antibiotics is not suggested. (2D)

The use of ACEI/ARB and diuretics should be temporarily discontinued during acute hemorrhage to prevent acute kidney injury. Most gross hematuria and cyst hemorrhage are self-limiting, and the bleeding can stop within 2-7 days. Patients with mild conditions should be placed on bed rest and drink more water (2-3 L/day). 
2. Patients with persistent hemorrhage for more than 1 week or patients over 50 years of age should undergo abdominal CT or MRI screening. (2D)

\subsubsection{Nephrolithiasis}

1. Unenhanced CT is the preferred imaging modality for the diagnosis and evaluation of suspected nephrolithiasis in patients with ADPKD. (1B)

Dual-energy CT can differentiate uric acid stones and calcium-containing stones.

2 We suggest that patients with ADPKD with nephrolithiasis drink more water (2-3 L/day). Three kidney stone types observed in patients with $\mathrm{ADPKD}$, i.e., uric acid stones, hypocitraturic calcium oxalate stones, and distal renal tubular acidification defects, can be treated with potassium citrate. (not graded)

3. Extracorporeal shock wave lithotripsy or percutaneous nephrolithotomy can be used according to the size and location of the stone in clinical circumstances. Ureterorenoscopy with laser fragmentation can also be used. (not graded)

\subsubsection{Renal Cyst Infection}

1. We suggest that 18 F-fluorodeoxyglucose-positron emission tomography be used to help with the diagnosis of cyst infections. (2D)

ADPKD patients with fever, abdominal pain, and an elevated erythrocyte sedimentation rate and C-reactive protein and procalcitonin levels should be screened for renal cyst infection. Escherichia coli is the most common pathogenic bacterium.

2. We suggest that molecular biological tests be used for the diagnosis of pathogenic microorganisms when blood and urine cultures are negative. (not graded)

3. We suggest that antibiotics be selected for anti-infection based on blood and urine culture results (if available). (2D)

Water-soluble antibiotics (cephalosporins, carbapenems, etc.) could be added to the treatment regimen for patients who do not exhibit improved symptoms after $72 \mathrm{~h}$ of treatment. Antibiotic treatment should last for at least 14 days or until the symptoms disappear, the body temperature returns to normal, and at least 2 negative blood or urine cultures are obtained.

\subsubsection{Chronic Pain}

1. We recommend that abdominal or back pain should be evaluated in patients with ADPKD during clinical visits. (1D)
2. We recommend a sequential medication approach according to the WHO pain relief ladder for patients with different levels of pain and renal function. (1D)

Nonopioid analgesics (such as acetaminophen) can be used as first-line analgesics; the long-term use of nonsteroidal anti-inflammatory drugs or COX-2 inhibitors is not recommended. Opioid analgesics can be considered if the above-mentioned drugs are ineffective or drug resistance occurs. Analgesic adjuvants such as gabapentin tablets and tricyclic antidepressants may have auxiliary effects.

3. Surgical treatments such as cyst sclerotherapy, laparoscopic decompression, and nephrectomy can be considered according to the size, number, and location of the cysts. (not graded)

A single cyst with a diameter $>5 \mathrm{~cm}$ or 3 or more cysts with a diameter $>4 \mathrm{~cm}$ can be treated by percutaneous cyst aspiration to reduce pain. Based on the level of pain relief achieved, further treatment of cyst sclerotherapy or laparoscopic cyst decompression can be considered. Celiac plexus block, radiofrequency ablation, percutaneous renal sympathetic nerve ablation, and spinal cord stimulation can also be considered to treat chronic pain.

\subsection{Treatment of End-Stage Renal Disease}

4.5.1 We recommend that kidney transplantation is the optimal choice of renal replacement therapy for appropriate patients with ESRD due to ADPKD. (1C)

4.5.2 We recommend that either hemodialysis or peritoneal dialysis is a suitable renal replacement therapy modality when kidney transplantation is not appropriate or for patients waiting for transplantation. (1C)

4.5.3 We suggest that nephrectomy should not be routinely performed prior to transplantation. (2C)

The indications for nephrectomy include recurrent or severe infection, symptomatic nephrolithiasis, intractable pain, recurrent or severe bleeding, space restrictions, and suspicion of renal tumor prior to transplantation. Laparoscopic nephrectomy is better tolerated than open surgery.

4.5.4 Therapeutic targets for hemoglobin, blood pressure, lipids, and anticoagulation are the same in patients with ADPKD compared to other patients with ESRD on dialysis. (not graded)

\subsection{Management of Intracranial Aneurysms}

4.6.1 We do not recommend widespread screening for intracranial aneurysms because most small intracranial aneurysms have a low risk of rupture and prophylactic repair may be risky. (2B)

4.6.2 We suggest screening for intracranial aneurysms in high-risk patients with ADPKD (with a positive family 
history of intracranial aneurysms or subarachnoid hemorrhage, previous intracranial aneurysm rupture, intracerebral hemorrhage, severe headache or neurological symptoms, and other situations if needed) (2B)

Patients with small, untreated unruptured intracranial aneurysms should be reevaluated at 6- to 24-month intervals. Patients with a family history of intracranial aneurysm and negative screenings should be rescreened every 5 years.

4.6.3 We recommend MRA or CTA as the screening method of intracranial aneurysms in patients with ADPKD. (1C)

4.6.4 We suggest that the treatment of intracranial aneurysms be discussed with a neurosurgeon at a center that has expertise in microsurgery and endovascular coiling. (2B)

\subsection{Management of PLD}

4.7.1 We recommend screening for PLD in patients with ADPKD using abdominal ultrasound at the initial assessment. (1C)

4.7.2 We recommend that females with ADPKD at risk of symptoms from PLD avoid estrogen supplements. (1D)

4.7.3 We suggest surgery and medication for patients with severe PLD whose symptoms severely affect their quality of life (abdominal pain, distension, early satiety, back pain, and gastroesophageal reflux). Surgical methods include aspiration, sclerotherapy, decompression, partial or segmental hepatectomy, and liver transplantation. (not graded)

4.7.4 We suggest PET-CT for the diagnosis of hepatic cyst infections. (not graded)

4.7.5 We suggest that treatment of hepatic cyst infections requires fluoroquinolone for at least 6 weeks. If fe- ver persists for more than $72 \mathrm{~h}$, third-generation cephalosporins should be used. For patients with persistent symptoms for 3-5 days, a PET-CT examination can be performed to determine the location of the infected cysts, followed by the puncture and drainage of the cysts and a drug susceptibility culture. (not graded)

\section{Chapter 5: Family Planning}

5.1 We suggest that patients with ADPKD who are planning a family receive genetic counselling and preimplantation genetic testing before pregnancy. (2D)

The use of preimplantation genetic testing to block the inheritance of ADPKD pathogenic gene mutations can reduce the birth rate of offspring with ADPKD. Currently, ADPKD inheritance has been successfully blocked in 30 families in China. The decisions should be made by the patients with ADPKD.

5.2 We suggest multiple annealing and looping-based amplification cycle technology combined with in vitro fertilization access as preimplantation genetic testing methods. (not graded)

\section{Disclosure Statement}

The authors have no conflict of interests to disclose.

\section{Funding Sources}

Not applicable. 